\title{
A New Image Inpainting Approach based on Criminisi Algorithm
}

\author{
Nouho Ouattara ${ }^{1}$, Georges Laussane Loum ${ }^{2}$, Ghislain Koffi Pandry ${ }^{3}$, Armand Kodjo Atiampo ${ }^{4}$ \\ Laboratoire de Recherche en Informatique et Télécommunication/UMRI EEA \\ Institut National Polytechnique Felix-Houphouët Boigny \\ Yamoussoukro, Côte d'Ivoire
}

\begin{abstract}
In patch-based inpainting methods, the order of filling the areas to be restored is very important. This filling order is defined by a priority function that integrates two parameters: confidence term and data term. The priority, as initially defined, is negatively affected by the mutual influence of confidence and data terms. In addition, the rapid decrease to zero of confidence term leads the numerical instability of algorithms. Finally, the data term depends only on the central pixel of the patch, without taking into account the influence of neighboring pixels. Our aim in this paper is to propose an algorithm to solve the problems mentioned above. This algorithm is based on a new definition of the priority function, a calculation of the average data term obtained from the elementary data terms in a patch and an update of the confidence term slowing its decrease and avoiding convergence to zero. We evaluated our method by comparing it with algorithms in the literature. The results show that our method provides better results both visually and in terms of the Peak Signal-to-Noise Ratio (PSNR) and Structural SIMilarity index (SSIM).
\end{abstract}

Keywords-Image inpainting; Criminisi algorithm; priority function; data term; confidence term; identity function

\section{INTRODUCTION}

Problems related to image degradation are numerous and occur in several areas. In the case of medical imaging, especially endoscopic imaging, some highlights may appear as white areas and this is often embarrassing when interpreting the scene [1]-[4]. This is similar to the search for white objects in a foliage. Here, the highlights are sometimes confused with the white objects being sought.

For the correcting of the above-mentioned problems, the technique of digital image inpainting is generally used. Image inpainting is intended to fill in missing parts of an image or to remove information that disturbs the interpretation of a scene. There are mainly two categories of inpainting methods: those based on diffusion processes and those based on exemplars [5]-[7].

Diffusion-based methods [8]-[11] simulate how human proceeds to fill a missing part in an image. These methods use the pixels of the known region neighboring the region to be restored to determine the structure and content of the diffusion. This reconstruction is done iteratively, from near to near, the outside to the inside of the unknown area. Masnou et al. [8] were the first to propose a diffusion-based inpainting model. Their disocclusion model is based on level lines. Subsequently, Bertalmio et al. [9] described a model based on partial difference equations (PDEs) called the BertalmioSapiro-Caselles-Bellester model (BSCB). Chan and Shen [10] developed a model by minimizing total variation (TV) using the Euler-Lagrange equation. In addition, in order to take into account geometric curves, they proposed the Curvature Diffusion model (CCD). The main limitation of diffusionbased inpainting methods is the size of the area to be restored. These methods provide good results for small areas. However, for large areas, they produce a blurred effect in painted areas and thus reduce the quality of the restoration.

To solve this problem, exemplar-based methods have been proposed [12]-[18]. These methods are based on repeating patterns in an image. Efros and Leung [19] were the first to present a model based on image statistics and similarity between different image regions. Criminisi et al. [12] have revolutionized inpainting methods based on pattern repetition by integrating textural and structural information. Their algorithm preserves textures and linear structures and provides better results than geometric methods for large areas.

However, for reasons of inconsistencies in restoration due to the parameters related to the Criminisi algorithm, several studies have been conducted to improve it.

Criminisi et al. proposed in their approach a priority function to define the filling order of the area to be reconstituted. This priority function is based on the multiplication of two terms, confidence and data. Work has been done to show the influence that each term can have on the other based on the product [13], [14]. Indeed, when one of the two terms tends to zero, the product with the second term gives a result of very low value. This can negatively affect the filling order and therefore the quality of the restoration. Thus, some researchers have proposed several methods to solve the problem related to the calculation of the priority function [20][24]. Chi et al. [20] proposed to raise the confidence term to a power of 3 in order to increase the importance of the data term. Their goal is to improve texture details to increase the accuracy of the restoration. A commonly used approach is to replace the multiplication with a weighted sum to avoid the influence of one term on the other [13]-[15].

Another major problem of Criminisi algorithm is the rapid convergence of the confidence term to zero when it is updated. Indeed, to favor pixels that have never been filled or that have been earlier, the value of the confidence term of the filled pixels is lower than those that allowed it to be updated. This convergence to zero can lead to poor restoration of large areas. 
Nan and Xi [13] then used a logistics function to update the confidence term for large areas. When the area to be restored contains less than 1500 pixels, the update proposed by [12] is used. Otherwise, they use the derivative of the logistics function. Unfortunately, the principle of reducing the value of the confidence term is not always respected. There is generally an increase in the value of the confidence term rather than a decrease in large areas. Yuheng and Hao [25] proposed redefining the confidence term to avoid its convergence to zero by combining Manhattan's distance with that proposed in the Criminisi algorithm.

Improvements in the priority function were also made to the data term. Hou [15] proposed a new approach of Criminisi algorithm by providing sequential structural optimization. $\mathrm{He}$ defined a new data term and used the Sobel gradient instead of the one used in Criminisi algorithm to strengthen the repair of highly structural regions. Wu and Ruan [26] exploited the TV diffuse model while Xi et al. [27] used entropy as a data term. Yin and Chang [28] have improved the data term by introducing isophote curvature information.

In order to avoid the problem of the multiplication of confidence and data terms in calculating the priority of [12], we propose to use their weighted sum. We also propose a new approach of confidence term update based on two linear functions to avoid convergence to zero. The first is lower than the identity function up to a defined threshold value. Its role is to promote patches that have never been filled. The second function is close to a constant. It allows to bring closer to the threshold, all the update values that are lower than it. Finally, to take into account all the structural information in a patch to be reconstructed, the value of the data term of each boundary pixel is calculated and their average value is retained as the value of the data term of this patch.

The rest of this paper is structured as follows. Section 2 is devoted to a detailed review of the Criminisi algorithm. In Section 3, we present our approach. Section 4 presents our results and a comparative analysis with those of the methods in the literature. Our work ends with a conclusion that leads to perspectives.

\section{CRIMINISI AlgORITHM}

The Criminisi algorithm is an algorithm capable of restoring linear structures and texture simultaneously. It is suitable for repairing large damaged surfaces. It assumes that an image to be restored consists of three parts. The first part is the known region $(\boldsymbol{\Phi})$, the second is the unknown region $(\Omega)$ and the third is the border separating the two regions $(\delta \Omega)$. This principle is illustrated in Fig. 1.

To fill the unknown area, some elements must be defined. The $\delta \Omega$ border is made up of a set of pixels. A pixel of this boundary is designated by p. $\psi_{p}$ is a patch to be reconstituted centered on the pixel p. It is also called the target block. This patch contains a known part and an unknown part that will be rebuilt. The gradient $\nabla \mathrm{I}_{\mathrm{p}}^{\perp}$ represents the intensity and direction of the illumination line and $\mathrm{n}_{\mathrm{p}}$ is the normal unit vector at the contour $\delta \Omega$ at point $\mathrm{p}$. The gradient $\nabla \mathrm{I}_{\mathrm{p}}^{\perp}$ is defined by (1).

$$
\nabla I_{p}^{\perp}=\frac{\left(-I_{y}(p), I_{x}(p)\right)}{\sqrt{\left[I_{x}(p)\right]^{2}+\left[I_{y}(p)\right]^{2}}}
$$

The Criminisi algorithm is described in four steps.

\section{A. Step 1: Calculate the Priorities of Each Patch Along the $\delta \Omega$ Border and Select the Patch with the Highest Priority}

The priority is used to define the filling order and the highest value determines the patch to be filled. The priority is calculated as follows (2):

$$
P(p)=C(p) D(p)
$$

where $\mathrm{C}(\mathrm{p})$ and $\mathrm{D}(\mathrm{p})$ are respectively the confidence term and the data term. These terms are defined as follows (3) and (4):

$C(p)=\frac{\sum_{q \in \Psi_{p} \cap \Phi} C(q)}{\left|\Psi_{p}\right|}$

$D(p)=\frac{\left|\nabla I_{p}^{\perp} \cdot n_{p}\right|}{\alpha}$

$\left|\Psi_{p}\right|$ represents the total number of pixels in the patch $\psi_{p}$ and $\alpha$ is a normalization coefficient. The value of $\alpha$ is $256 \mathrm{x}$ 3 for an 8-bit RGB image.

The confidence term must follow the initial conditions defined in (5):

$$
C(q)= \begin{cases}0 & \text { if } q \in \Omega \\ 1 & \text { if } q \in \Phi\end{cases}
$$

The selected patch is obtained from (6):

$$
\Psi_{\hat{p}}=\operatorname{Arg} \max _{\Psi_{p}} P(p)
$$

\section{B. Step 2: Determination of the Best Filling Patch}

The search for the best patch corresponding to the patch to be filled is carried out in the known region. The selected patch is obtained by comparing the known part of the patch to be inpainted with its correspondent in the filling patch. This comparison is made through the sum of squared differences (SSD). The SSD is defined by (7):

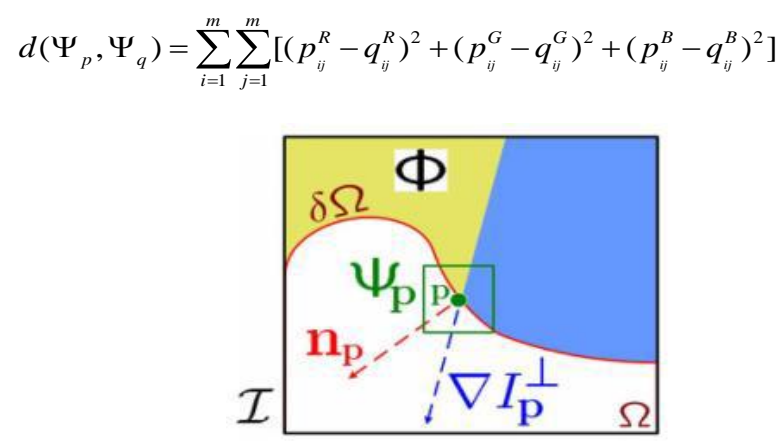

Fig. 1. Basic Principle of Criminisi Algorithm. 
where $p_{i j}^{R}, p_{i j}^{G}$ and $p_{i j}^{B}$ are the RGB color channel values of the pixels of the patch to be filled and $q_{i j}^{R}, q_{i j}^{G}$ and $q_{i j}^{B}$ are those of the current patch. $m$ is the number of known pixels in the $\psi_{p}$ patch to be restored. The selected patch is the one with a minimum $d\left(\psi_{p}, \psi_{q}\right)$ value. Its equation is as follows (8):

$\Psi_{\hat{q}}=\operatorname{Arg} \max _{\Psi_{q} \in \Phi} d\left(\Psi_{\hat{p}}, \Psi_{q}\right)$

The filling process is illustrated in Fig. 2 .

In Fig. 2(a), $\psi_{q}$ is selected as the patch similar to $\psi_{p}$. Thus, in Fig. 2(b), its content is used to fill in the unknown part of $\psi_{p}$. The inpainting result is perceived in Fig. 2(c).

\section{Step 3: Update of the Confidence Term}

When a patch is filled with the pixel values of the best corresponding patch, the pixels of this patch initially belonging to the unknown part of the image, will have a new value of the confidence term. This value is that of the central pixel of the patch calculated from (3). The expression of the confidence term update is defined as follows (9):

$$
C(q)=C(\hat{p}) \quad \forall q \in \Psi_{\hat{p}} \cap \Omega
$$

D. Step 4: Repeat Steps 1 to 3 until the Area to be Inpainted is Completely Filled

\section{PRoposed Method}

\section{A. Improvement of the Priority Function and Data Term}

Several authors have shown that the priority function proposed in the Criminisi algorithm using the product of confidence and data terms, negatively influences the filling order and therefore, the result of the restoration. Thus, an alternative that has addressed the problem of the multiplication of confidence and data terms is the use of the weighted sum. Until now, the calculation of the filling priority has been based on the central pixel of the patch to be restored.

However, a patch can be defined as a sub-image contained in the entire image I. In this case, a patch to be inpainted $\psi_{p}$ contains three parts: a known part $\psi_{p} \cap \Phi$; an unknown part $\psi_{p} \cap \Omega$; and a set of boundary pixels $\psi_{p} \cap \delta \Omega$. Fig. 3 shows an image in which a patch to be inpainted is defined.

In Fig. 3, the red line in the black contour ellipse represents all the boundary pixels of the patch to be filled $\psi_{p}$. Thus, we believe that each pixel of this boundary can provide information about the data term.

In our approach, we propose a new priority function based on all the pixels of $\psi_{p} \cap \delta \Omega$ and not only on the central pixel of the patch. This function also exploits the advantages of the weighted sum of the confidence and data terms. Its formula is defined by (10):

$$
\begin{aligned}
& P(p)=\alpha C(p)+\beta \bar{D}(p) \\
& \quad \text { with } \\
& \alpha+\beta=1
\end{aligned}
$$

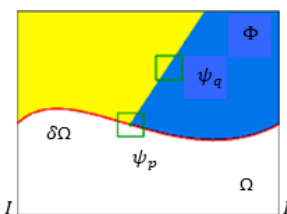

(a)

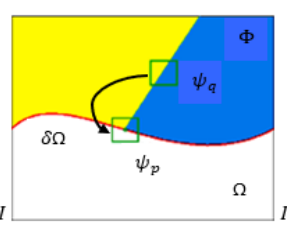

(b)

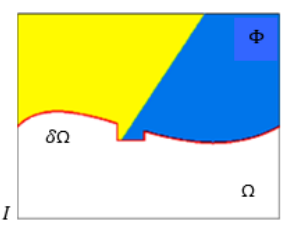

(c)
Fig. 2. Patch Filling Process.

where the term $\bar{D}(p)$ is the average value of the data terms defined for each pixel of the border between known and unknown regions in the patch $\psi_{p}$. It is obtained from (12):

$\bar{D}(p)=\frac{\sum_{p \in \delta \Omega \cap \Psi_{p}} D(p)}{\left|\delta \Omega \cap \Psi_{p}\right|}$

\section{B. Proposition to update the Confidence Term}

1) Motivation and general description of our method: In order to favor patches that have never been filled or patches that have been filled earlier, Criminisi et al. proposed to reduce the value of the updated confidence term of the newly filled pixels compared to the confidence term values of the known pixels. As a result, after several filling levels, the values of the updated confidence terms decrease to zero. Their convergence is a limitation to their algorithm. This has been the subject of studies by several researchers who have tried to solve this problem [12],[23].

To address the problem of convergence of confidence terms to zero, we propose to slow the decrease of its update. Slowing down the decrease of the confidence term does not guarantee non-convergence to zero. Thus, we define a threshold value from which we will increase the $C(\hat{p})$ value of the obtained confidence term.

Indeed, after a filling, the value of the confidence term of the patch to be filled $C(\hat{p})$ can be either lower or higher than the initial minimum value of the confidence terms. In the case where this value is higher than the initial minimum, the update value of the confidence term must decrease but remain higher than the initial minimum. Otherwise, the updated confidence term must increase while remaining below the initial minimum. This double adjustment makes it possible to guarantee the decrease in confidence terms and to avoid convergence to zero. Fig. 4 illustrates our model of confidence term update.

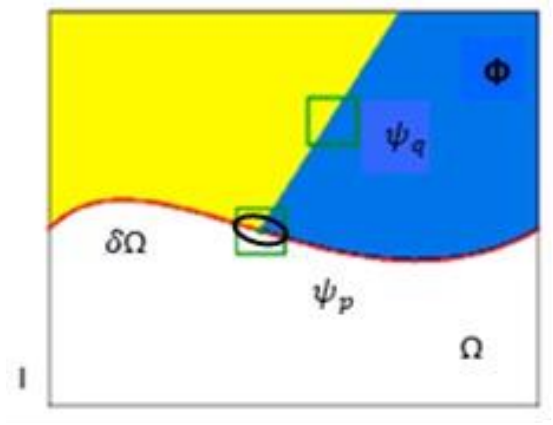

Fig. 3. Representation of a Patch to be Inpainted. 


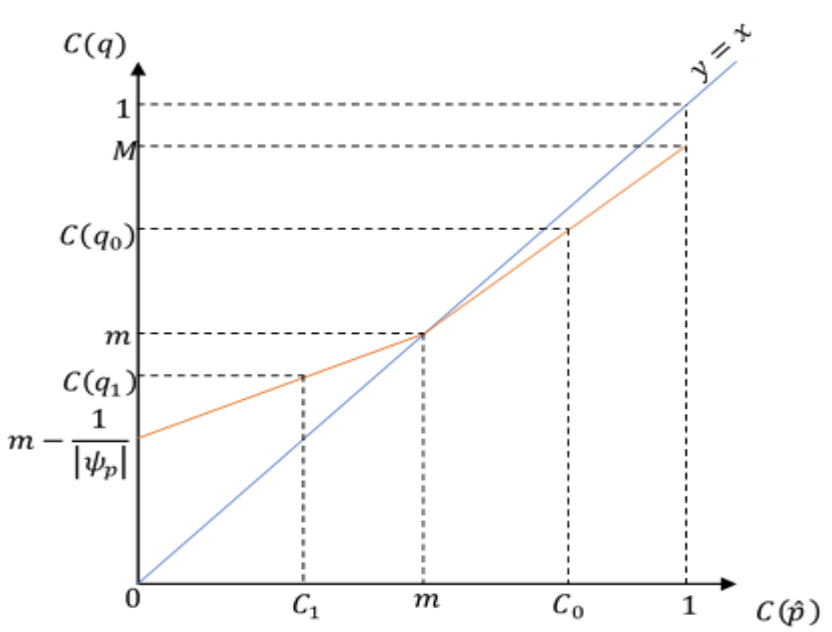

Fig. 4. Evolution of the Confidence Term update C(q).

The blue line represents the identity function as well as the update of the Criminisi confidence term. The two segments in red are a representation of our update of the confidence term. $\mathrm{M}$ and $\mathrm{m}$ are respectively the maximum and minimum values of the confidence term of the $\delta \Omega$ border pixels in the initial image. The number $\frac{1}{\left|\psi_{p}\right|}$ is a very small value to control the convergence of the updated confidence term $\mathrm{C}(\mathrm{q})$ when the value of the confidence term of the reconstituted patch is less than $\mathrm{m}$. $\mathrm{q}$ is a pixel of the initially unknown area of the patch that has just been filled. $\mathrm{C}_{0}$ and $\mathrm{C}_{1}$ are values of the confidence terms of the patches filled in $C(\hat{p}) . C\left(q_{0}\right)$ and $C\left(q_{1}\right)$ are their updated correspondents $\mathrm{C}(\mathrm{q})$ respectively. We can see that $C\left(q_{0}\right)$ is less than $\mathrm{C}_{0}$ and $C\left(q_{1}\right)$ more than $\mathrm{C}_{1}$. $\mathrm{C}(\mathrm{q})$

2) Steps of the algorithm of the confidence term update

- Calculation of the confidence terms of the patches to be reconstituted (confidence term of the central pixel) at initialization: $C_{0}(p)$;

- Search for the minimum and maximum of the initial $\mathrm{C}(\mathrm{p}): \mathrm{m}=\min \left(C_{0}(p)\right)$ and $\mathrm{M}=\max \left(C_{0}(p)\right)$;

- Search for two linear functions to simulate the evolution of confidence terms:

When the confidence term of the filled patch $C(\hat{p})$ is between $\min \left(C_{0}(p)\right)$ and $\max \left(C_{0}(p)\right)$, the function must respect the constraint of reducing the value of the confidence term and the constraint of non-convergence to zero. To do this, we propose an increasing line that is below the first bisector $(\mathrm{y}=\mathrm{x})$ and cuts the first bisector in $\min \left(C_{0}(p)\right)$. This requires that the updated values $C(q)$ be between min $\left(C_{0}(p)\right)$ and $\max \left(C_{0}(p)\right)$. Thus, the linear function is defined from (13):

$C(q)=\alpha C(\hat{p})+\beta$

(13) with the following condition:

$C(q)= \begin{cases}\max \left(C_{0}(p)\right) & \text { if } C(\hat{p})=1 \\ \min \left(C_{0}(p)\right) & \text { if } C(\hat{p})=\min \left(C_{0}(p)\right)\end{cases}$ thus:

$\alpha=\frac{\max \left(C_{0}(p)\right)-\min \left(C_{0}(p)\right)}{1-\min \left(C_{0}(p)\right)}$

and

$\beta=\frac{\min \left(C_{0}(p)\right)\left[1-\max \left(C_{0}(p)\right)\right]}{1-\min \left(C_{0}(p)\right)}$

In a patch whose elementary confidence terms $\mathrm{C}(\mathrm{p})$ have values close to min $\left(C_{0}(p)\right)$, it is possible to obtain a confidence term of the patch to be reconstituted $C(\hat{p})<\min$ $\left(C_{0}(p)\right)$. Thus, the second function must be increasing. It must converge towards $\min \left(C_{0}(p)\right)$ and its graphical representation must be above the first bisector. Its growth must be slow. We define this function by a linear function verifying the conditions (17) and (18):

$$
\begin{aligned}
& C(q)=\min \left(C_{0}(p)\right)-\frac{1}{\left|\Psi_{p}\right|}, \quad \text { if } C(\hat{p})=0 \\
& C(q)=\min \left(C_{0}(p)\right), \quad \text { if } C(\hat{p})=\min \left(C_{0}(p)\right)
\end{aligned}
$$

The linear function thus obtained is as follows (19):

$$
C(q)=\frac{1}{\left|\Psi_{p}\right| \min \left(C_{0}(p)\right)} C(\hat{p})+\min \left(C_{0}(p)\right)-\frac{1}{\left|\Psi_{p}\right|}
$$

\section{EXPERIMENTAL RESULTS AND DISCUSSIONS}

\section{A. Choice of Parameters}

This section focuses on the choice of weighting coefficients for confidence and data terms and patch size. These choices are made concurrently. The experiments were carried out on a set of $256 \times 256$ colour images.

To better characterize the texture present in our images, we varied the window from $5 \times 5$ to $11 \times 11$ in increments of 2 . The variations in the weighting coefficient of the confidence term $\alpha$ were studied by varying it from 0.1 in the range 0.1 to 0.9 . Thus, for a given image, we obtain 36 images of which we must select the best one. This allows to choose both the best patch size and the best coefficients $\alpha$ and $\beta$ for the confidence and data terms respectively. Fig. 5 illustrates this experience.

The performance of the algorithms was evaluated using the Peak Signal-to-Noise Ratio (PSNR) and the Structural SIMilarity (SSIM). For an original image $I_{0}$ and the reconstructed image $I_{r}$, these two metrics are defined in (20) and (22).

$P S N R=10 \cdot \log _{10}\left(\frac{d^{2}}{M S E}\right)$

where $d$ is the dynamics of the original image and MSE is the Mean Square Error. This error is obtained by (21):

$$
M S E=\frac{1}{m n} \sum_{i=0}^{m-1} \sum_{j=0}^{n-1}\left(I_{o}(i, j)-I_{r}(i, j)\right)^{2}
$$



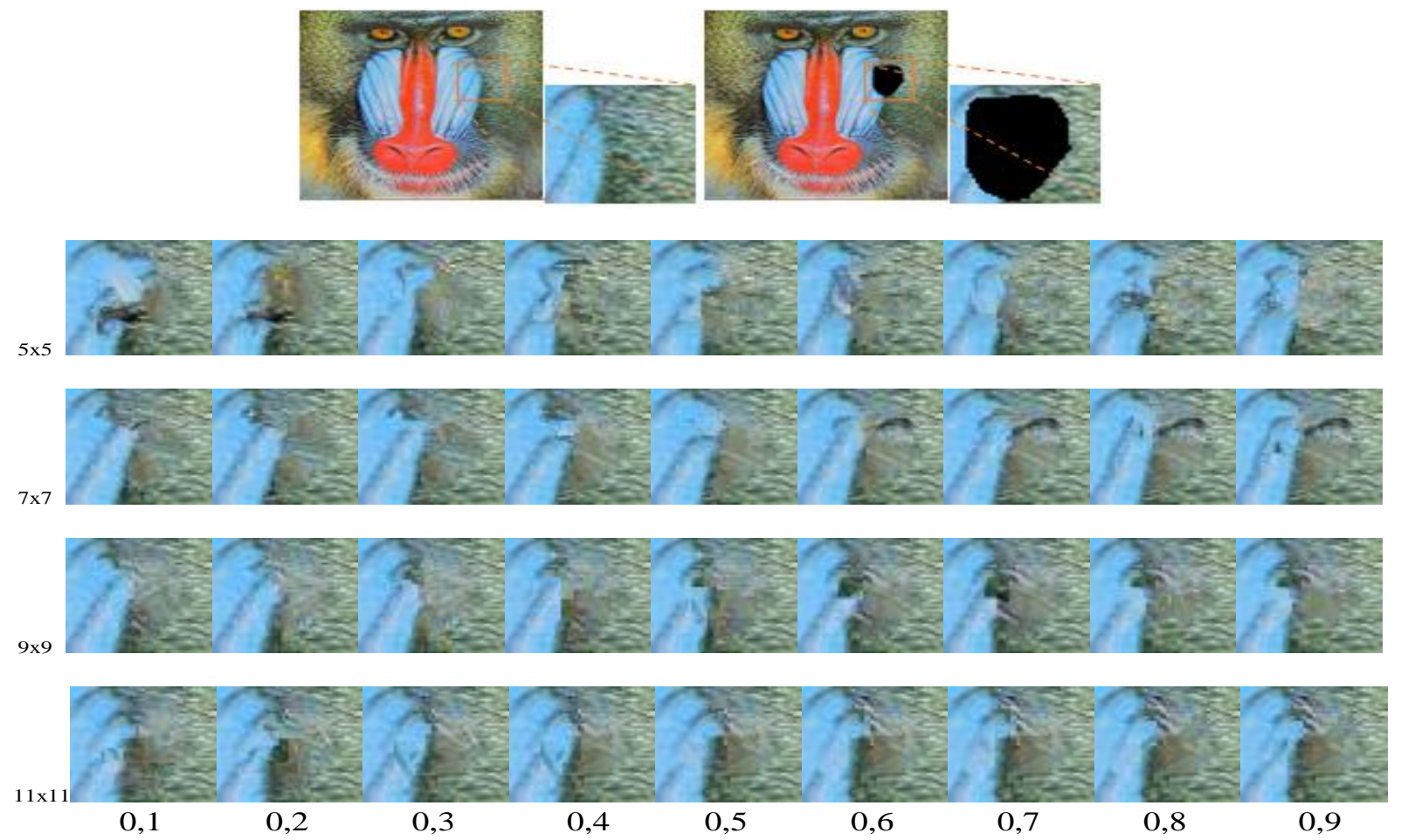

Fig. 5. Images Results of the Baboon Image Inpainting for different Patch Sizes and different Coefficients $\alpha$ of the Confidence Term.

with $\mathrm{m}$ and $\mathrm{n}$ denoting the number of rows and columns of the images $I_{0}$ and $I_{r}$.

$$
\operatorname{SSIM}(x, y)=\frac{\left(2 \mu_{x} \mu_{y}+c_{1}\right)\left(2 \sigma_{x} \sigma_{y}+c_{2}\right)\left(2 \operatorname{cov}_{x y}+c_{3}\right)}{\left(\mu_{x}^{2}+\mu_{y}^{2}+c_{1}\right)\left(\sigma_{x}^{2}+\sigma_{y}^{2}+c_{2}\right)\left(\sigma_{x} \sigma_{y}+c_{3}\right)}
$$

with

$-x$ and $y$ the original $I_{0}$ and restored $I_{r}$ images respectively;

- $\mu_{x}, \mu_{y}$ the respective averages of the images $x$ and $y$;

- $\sigma_{x}^{2}, \sigma_{y}^{2}$ their variance;

- $\operatorname{cov}_{x y}$ the covariance of $x$ and $y$;

- $c_{1}, c_{2}$ and $c_{3}$ the division stabilization variables when the denominator is very low.

The PSNR is used to measure the reconstitution consistency between the restored image and the original image. The SSIM allows to evaluate the visual quality of restoration by comparing the restored image and the original image.

These results show that it is difficult to define the best patch size and coefficient of the confidence term. However, the $9 \times 9$ and $11 \times 11$ size patches seem to produce the best results. For these patch sizes, coefficients 0.1 to 0.5 give better results. Starting from the observation that the visual aspect alone does not allow us to choose our parameters, we used PSNR and SSIM for the quantitative evaluation. The results are collected in Tables I and II.
TABLE. I. Baboon IMAge PSNR RESUlts For DifFERENT Patch Sizes AND VARIABLE COEFFICIENTS

\begin{tabular}{|l|l|l|l|l|}
\hline$\alpha$ & $5 \times 5$ & $7 \times 7$ & $9 \times 9$ & $11 \times 11$ \\
\hline 0,1 & 32,6694 & 35,9045 & 36,215 & 35,8816 \\
\hline 0,2 & 34,2809 & 35,3802 & 36,0053 & 35,5391 \\
\hline 0,3 & 36,2197 & 35,8309 & $\mathbf{3 6 , 9 1 9}$ & 36,2681 \\
\hline 0,4 & 34,913 & 36,2447 & 36,8832 & 36,2214 \\
\hline 0,5 & 34,932 & 35,8147 & 36,3861 & 35,7203 \\
\hline 0,6 & 35,3008 & 35,3759 & 34,6359 & 35,0639 \\
\hline 0,7 & 35,7355 & 35,2724 & 35,3292 & 35,2091 \\
\hline 0,8 & 34,1555 & 35,1694 & 35,6763 & 35,1676 \\
\hline 0,9 & 34,3502 & 35,3179 & 35,6818 & 35,3486 \\
\hline
\end{tabular}

TABLE. II. BABOON IMAGE SSIM RESUlTS FOR DIFFERENT PATCH SiZES AND VARIABLE COEFFICIENTS

\begin{tabular}{|l|l|l|l|l|}
\hline$\alpha$ & $5 \times 5$ & $7 \times 7$ & $9 \times 9$ & $11 \times 11$ \\
\hline 0,1 & 0,9909 & 0,9923 & $\mathbf{0 , 9 9 3}$ & 0,9916 \\
\hline 0,2 & 0,9903 & 0,9922 & 0,9928 & 0,9917 \\
\hline 0,3 & 0,9929 & 0,9923 & 0,9926 & 0,9926 \\
\hline 0,4 & 0,9911 & 0,9922 & 0,9926 & 0,9922 \\
\hline 0,5 & 0,9917 & 0,9926 & 0,9923 & 0,9914 \\
\hline 0,6 & 0,9913 & 0,9921 & 0,991 & 0,9909 \\
\hline 0,7 & 0,9916 & 0,9922 & 0,9912 & 0,991 \\
\hline 0,8 & 0,9912 & 0,9921 & 0,9917 & 0,9909 \\
\hline 0,9 & 0,9916 & 0,9922 & 0,9917 & 0,9911 \\
\hline
\end{tabular}


TABLE. III. BEST RESULTS F PSNR AND SSIM FOR DIFFERENT IMAGES AND DIFFERENT PATCH SIZES

\begin{tabular}{|c|c|c|c|c|c|}
\hline & & $5 \times 5$ & $7 \times 7$ & $9 \times 9$ & $11 \times 11$ \\
\hline & PSNR & 45,3101 & 45,5046 & 45,918 & 46,3161 \\
\hline & SSIM & 0,997 & 0,9973 & 0,9974 & 0,9973 \\
\hline & PSNR & 36,2197 & 35,9045 & 36,919 & 36,2681 \\
\hline & SSIM & 0,9929 & 0,9926 & 0,9930 & 0,9926 \\
\hline & PSNR & 33,4775 & 32,2674 & 33,9514 & 34,2936 \\
\hline & SSIM & 0,9809 & 0,981 & 0,9818 & 0,9808 \\
\hline & PSNR & 47,4129 & 47,6068 & 47,5378 & 47,1245 \\
\hline & SSIM & 0,9972 & 0,9975 & 0,9973 & 0,9972 \\
\hline & PSNR & 41,7133 & 44,2778 & 43,4745 & 43,764 \\
\hline & SSIM & 0,9969 & 0,9981 & 0,9978 & 0,9975 \\
\hline
\end{tabular}

In the PSNR table, the best result is obtained from the coefficient $\alpha=0.3$ and the size of the patch 9x9. On the other hand, in the SSIM, the resulting image by applying the coefficient $\alpha=0.1$ and the patch size 9x9 give the best result. This study was carried out on a set of images with different textures and structures. The best results of PSNR and SSIM for the different patch sizes are summarized in Table III.

This table shows that the best results, for images with more structure, are obtained from 9x9 patch sizes. This is perceptible through the three images at the top of Table III. For images containing more homogeneous areas, the $7 \times 7$ size provides the best results (see the two images at the bottom of Table III).

Thus, for the choice of the $\alpha$ coefficient, we have classified our set of images into two categories according to their textural and structural characteristics. We applied 9x9 size patches to six images containing textured, structured and homogeneous areas. We used $7 \times 7$ size patches of five other images containing areas that tended to be homogeneous. We varied the $\alpha$ coefficient from 0.1 to 0.9 and presented the results in Fig. 6 and 7. Fig. 6(a) and 7(a) represent the PSNR results obtained by applying $7 \times 7$ and $9 \times 9$ patches respectively according to the structural information of the images. The SSIM ones are represented by Fig. 6(b) and 7(b).

Through the results of the PSNR and SSIM, the choice of the best coefficient remains difficult. However, the 0.5 coefficient seems to be close to the best for $7 \times 7$ size patches and 0.4 for $9 \times 9$ size patches. Thus, we opt for the choice of $\alpha$ $=0.5$ and the $7 \times 7$ size patch when the area to be restored has fewer textures and structures. Otherwise, we choose $\alpha=0.4$ and the patch size $9 \times 9$. This imposes the values of 0.5 and 0.6 for $\beta$ respectively (11).

\section{B. Results and Evaluations}

In practice, it is difficult to quantitatively evaluate the result of image restoration using an inpainting method. Indeed, to perform the quantitative evaluation, it is necessary to know the original image that has not been damaged in order to determine the similarity with the restored image. Thus, our experiments will be carried out in two phases. The first concerns a set of images for which the information in the background of the area to be reconstructed is unknown. Fig. 8 and 9 illustrate this experience. In these cases, it is a subject of object removal. The evaluation is visual and therefore subjective. For an objective evaluation, we carried out the second phase of our experiments. We have selected other images in which we have inserted a task to remove. Fig. 10 to 17 present the results of this experiment. In Fig. 10 to 12, we also represented the evolution curves of the confidence terms and priority functions according to the number of iterations to show the influence of the product and the weighted sum on the evolution of the priority function. These curves also show the influence of the evolution of the confidence term on the shape of the priority function. We used the PSNR and SSIM to conduct the quantitative evaluation.

Table IV represents the comparative results of our algorithm with those of Criminisi and Nan.

\section{Discussions}

Fig. 8(c) to 8(e) are the inpainting results of the Criminisi, Nan and our algorithm respectively. The sections framed in red show the discussion areas of the three methods. We can see reconstruction defects at the bottom of the roof. In the Criminisi and Nan results, the defects are reflected in the appearance of certain plants on the roof. In our results, we can see a better reconstitution of the roof. The reconstruction of the shoreline seems to be of good quality for both our method and Nan's method, unlike Criminisi's result. In Fig. 9, we see a big difference in the background restoration. Our method seems to better reconstruct the information in the background of the removed tree. In the Criminisi and Nan results, however, there are abnormal earth blocks in the background of the tree.

The graphs obtained in Fig. 10(f), 11(f) and 12(f) show the rapid decrease in the confidence term proposed by Criminisi. This decrease, combined with the use of the product of the confidence and data terms, justifies the near-zero values of the priority function observed in Fig. 10(g), 11(g) and 12(g). This makes it difficult to determine the priority patch. Similarly, at the level of the algorithm proposed by Nan, the values of the confidence term are almost constant per interval or increase per place. This is due to the shape of an inverted parabola of the curve of the update confidence term. In the case where the values of the confidence term are constant, only the data term influences the priority function. This causes the observed oscillations around the same value of the priority function. This can have a negative impact on the reconstitution of textures. 


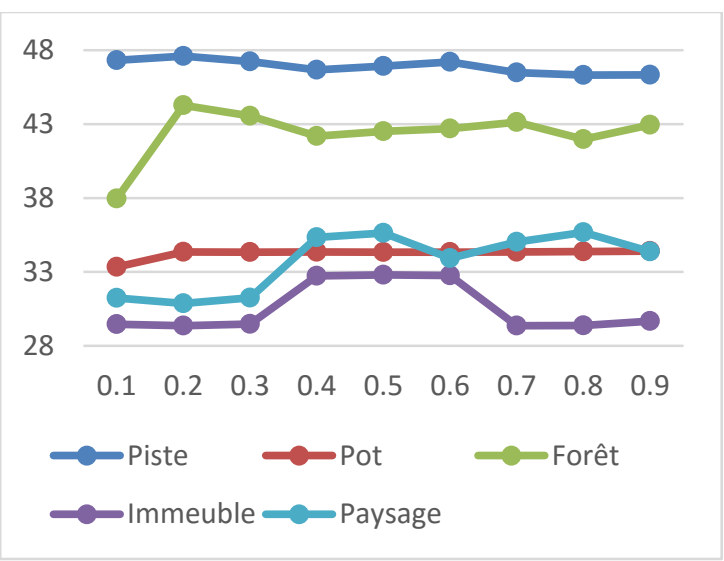

(a)

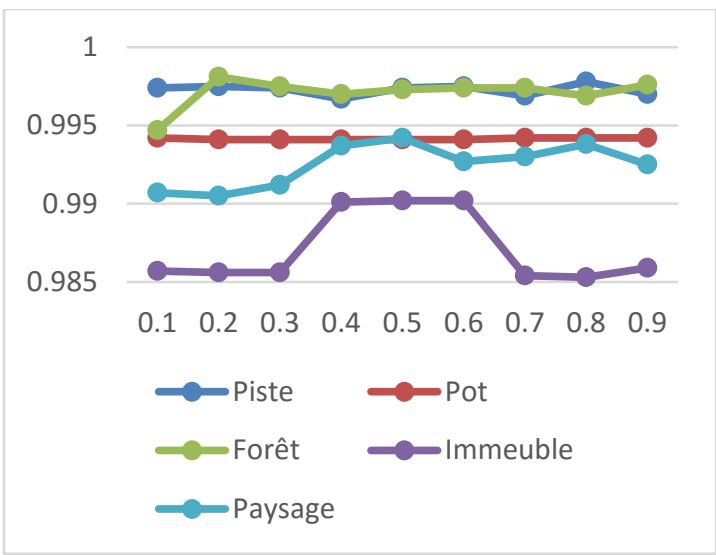

(b)

Fig. 6. Representation of PSNR and SSIM for different Coefficients $\alpha$ for 7x7 size Patches. (a) PSNR Graphs; (b) SSIM Graphs.

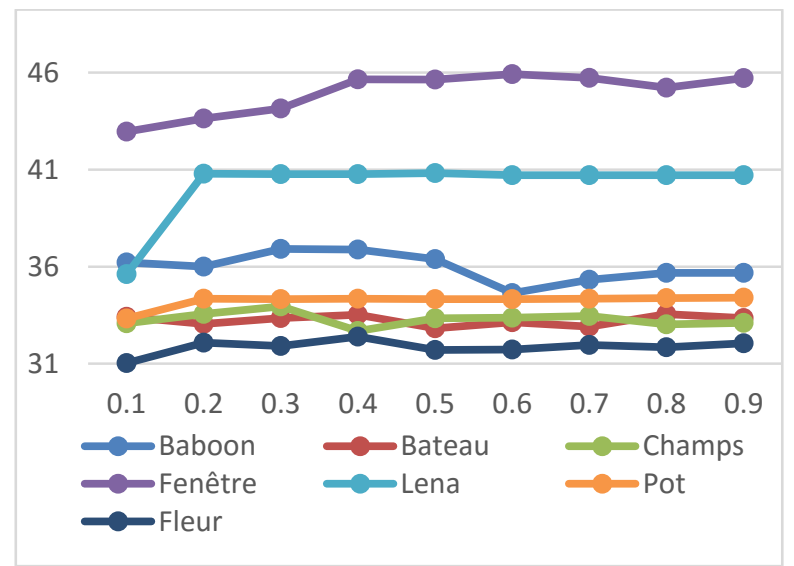

(a)

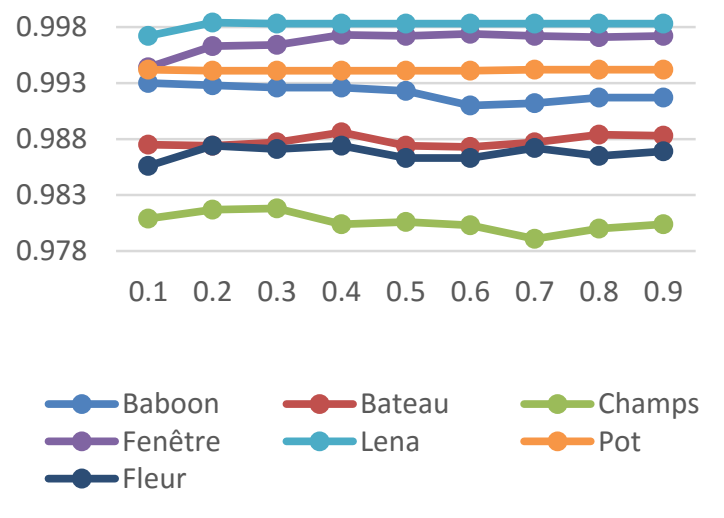

(b)

Fig. 7. Representation of PSNR and SSIM for different Coefficients $\alpha$ for 9x9 Size Patches. (a) PSNR Graphs; (b) SSIM Graphs.

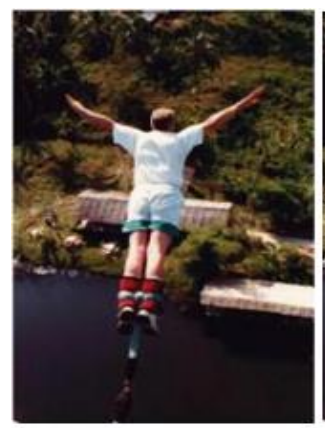

(a)

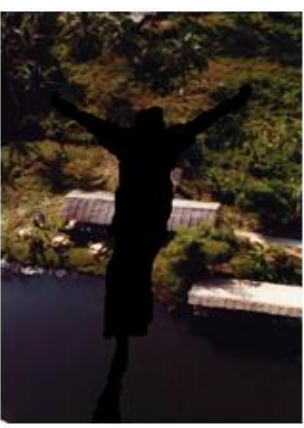

(b)

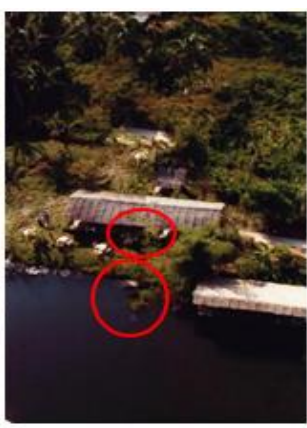

(c)

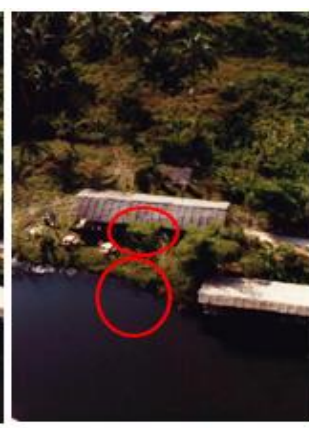

(d)

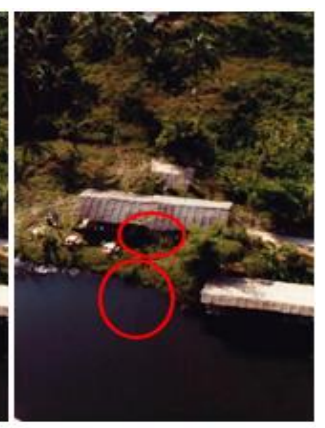

(e)

Fig. 8. Inpainting Result of "Bungee”. (a) Original Image; (b) Masked Image; (c) Criminisi Result; (d) Nan Result; (e) our Result.

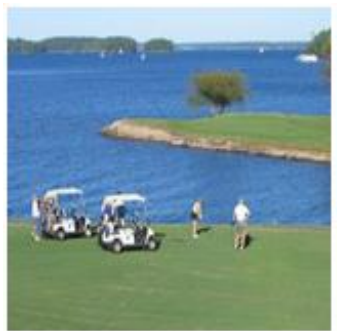

(a)

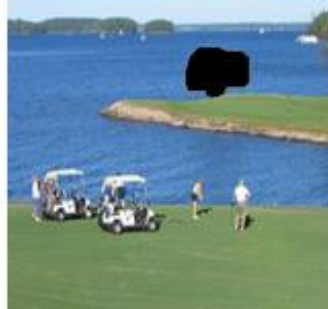

(b)

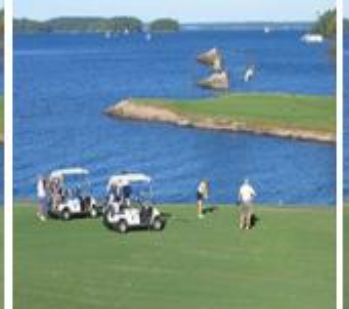

(c)

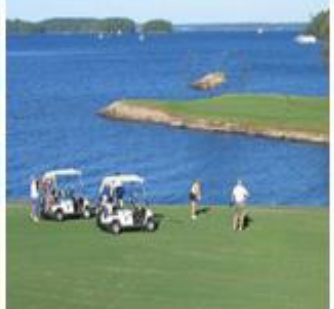

(d)

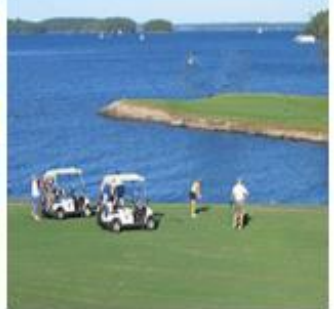

(e)

Fig. 9. Inpainting Result of "Island". (a) Original Image; (b) Masked Image; (c) Criminisi Result; (d) Nan Result; (e) Our Result. 


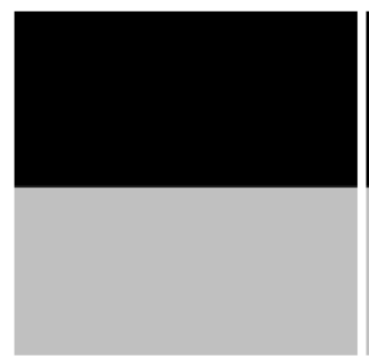

(a)

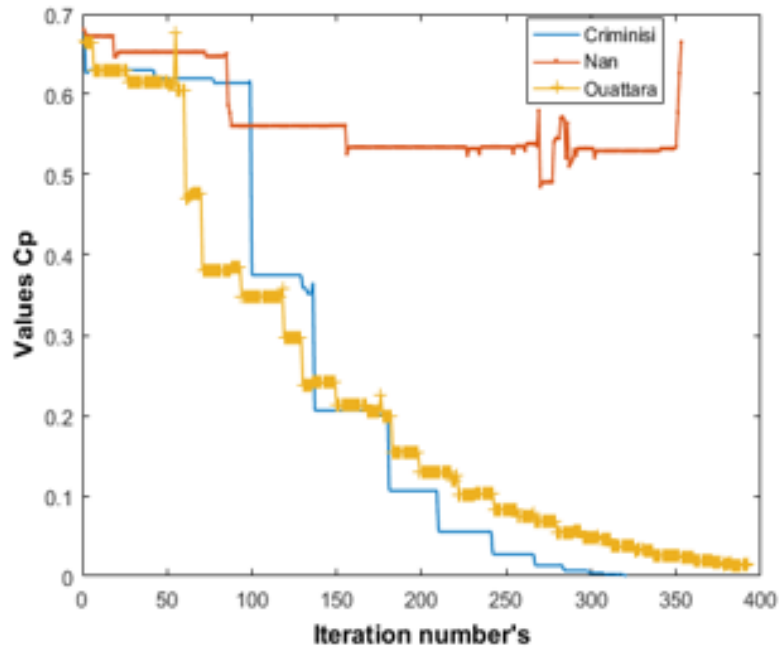

(f)

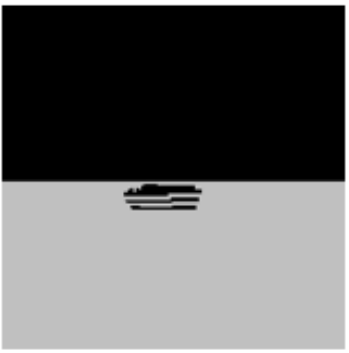

(c)

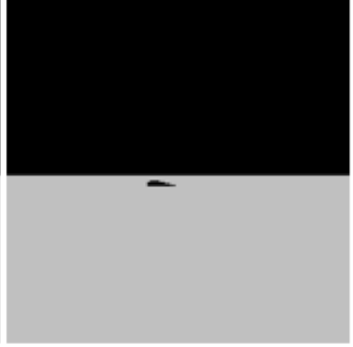

(d)

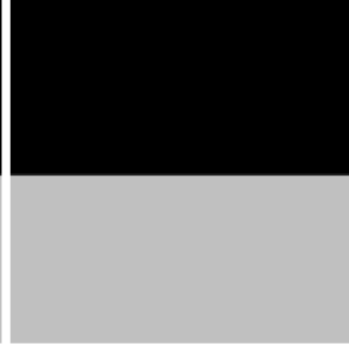

(e)

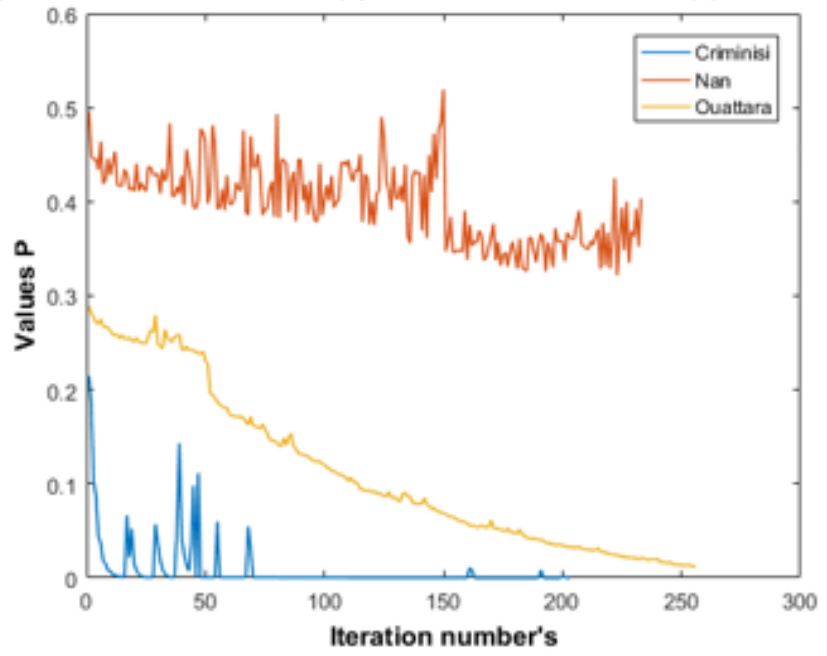

(g)

Fig. 10. Synthesis Image Inpainting Results. (a) Original Image; (b) Masked Image; (c) Criminisi Result; (d) Nan Result; (e) Our Result; (f) C(p) Values; (g) P(p) Values.

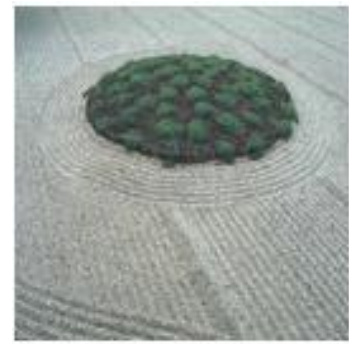

(a)

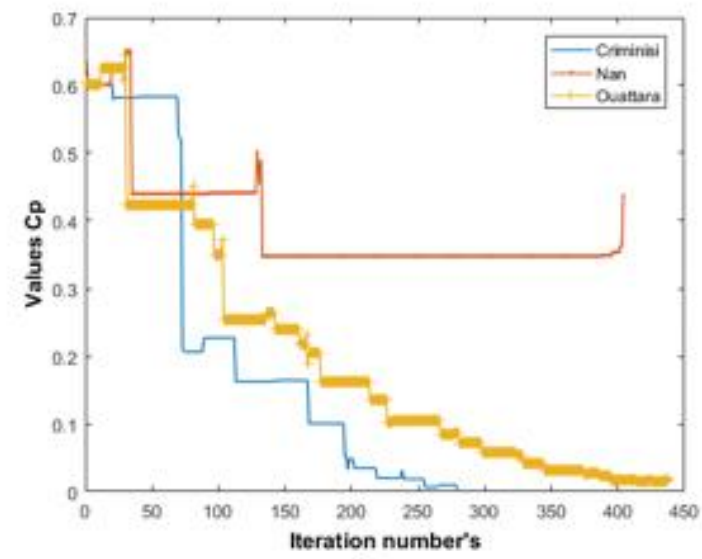

(f)

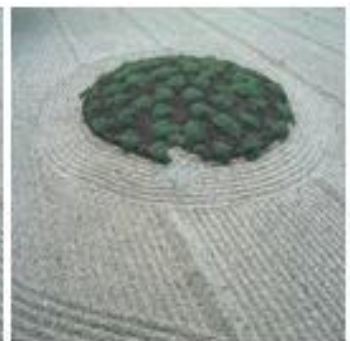

(c)

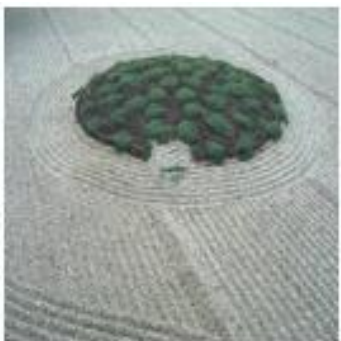

(d)

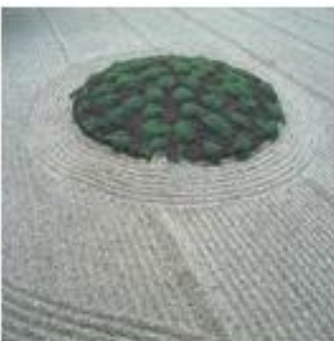

(e)

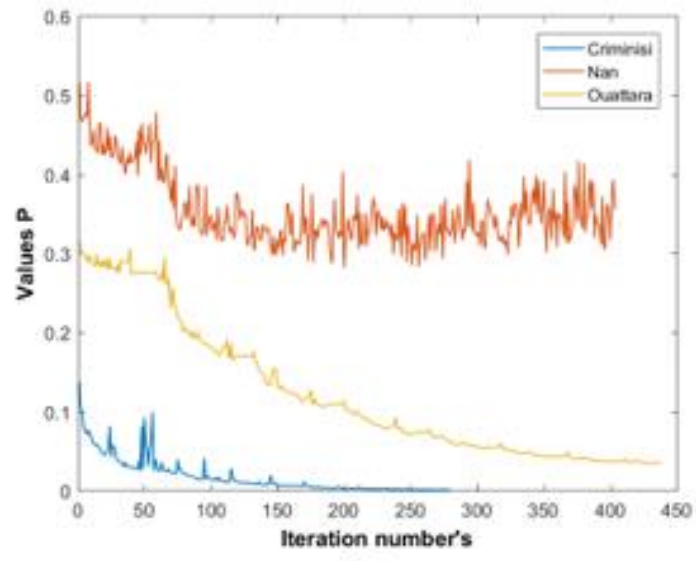

(g)

Fig. 11. Inpainting Result of "Nursery Garden”. (a) Original Image; (b) Masked Image; (c) Criminisi Result; (d) Nan Result; (e) Our Result; (f) C(p) Values; (g) P(p) Values. 


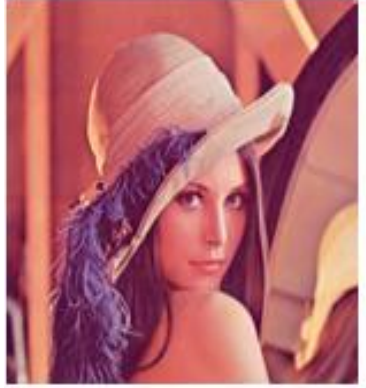

(a)

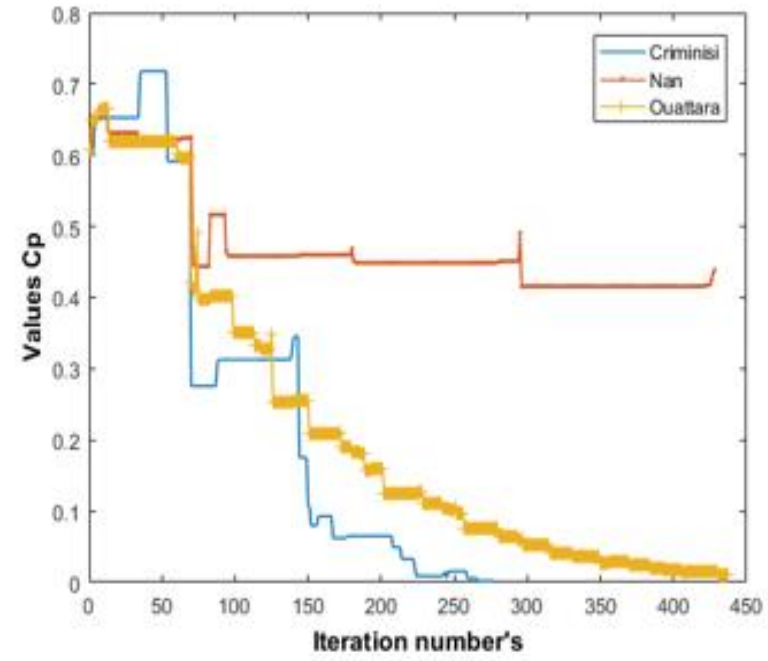

(f)

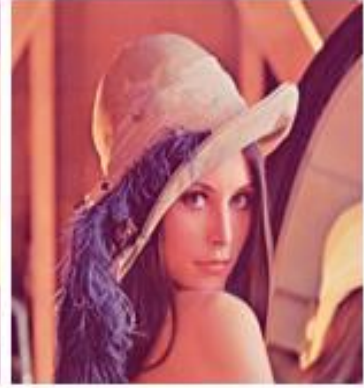

(c)

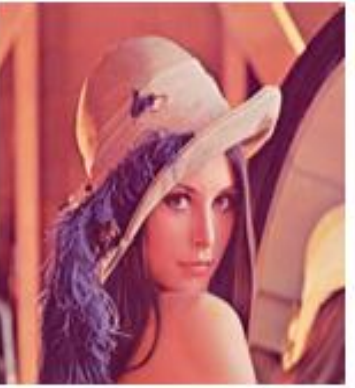

(d)

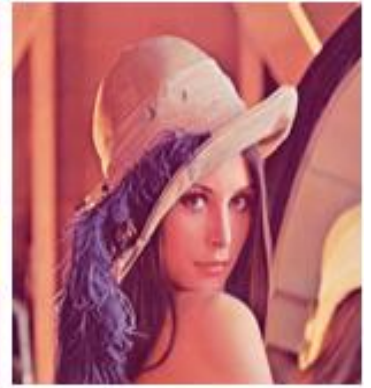

(e)

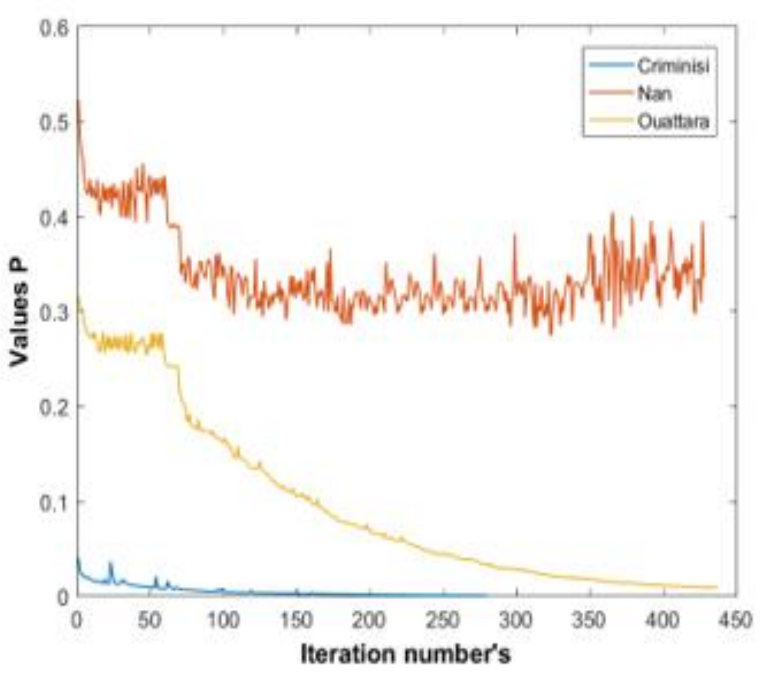

(g)

Fig. 12. Inpainting Result of "Lena". (a) Original Image; (b) Masked Image; (c) Criminisi Result; (d) Nan Result; (e) Our Result; (f) C(p) Values; (g) P(p) Values.

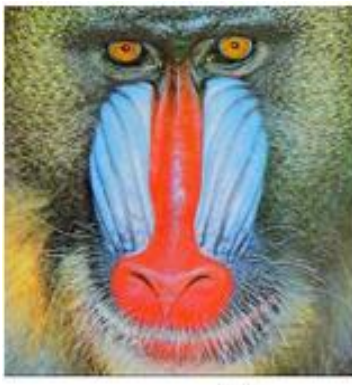

(a)

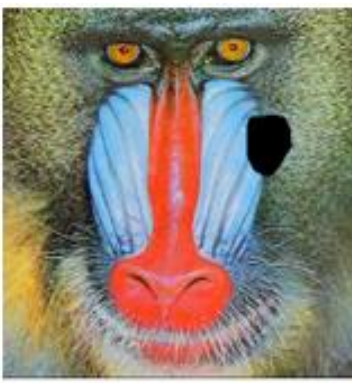

(b)

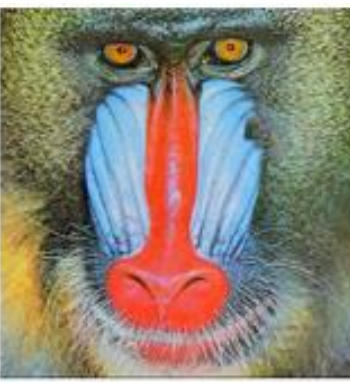

(c)

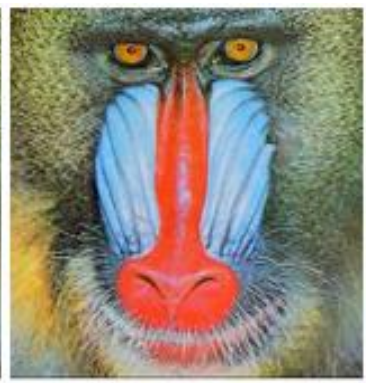

(d)

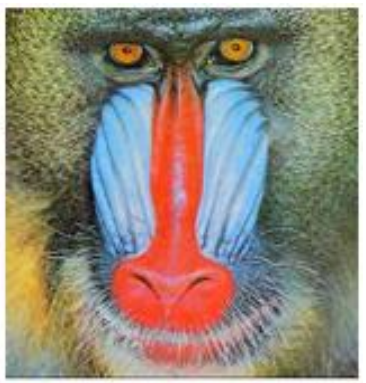

(e)

Fig. 13. Inpainting Result of "Baboon”. (a) Original Image; (b) Masked Image; (c) Criminisi Result; (d) Nan Result; (e) Our Result.

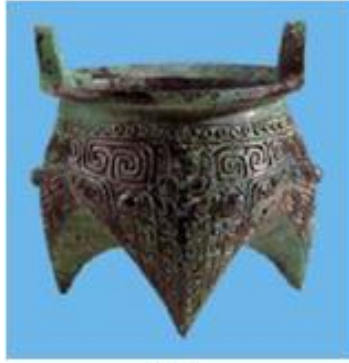

(a)

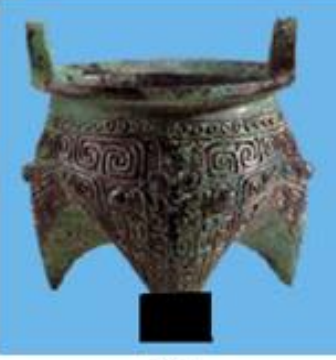

(b)

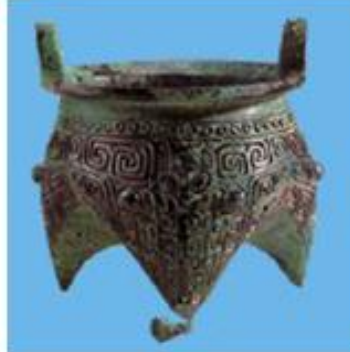

(c)

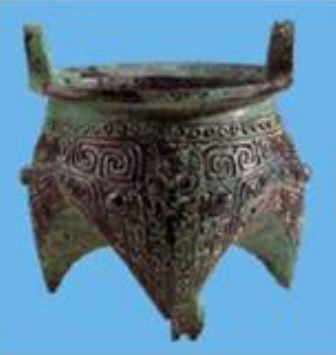

(d)

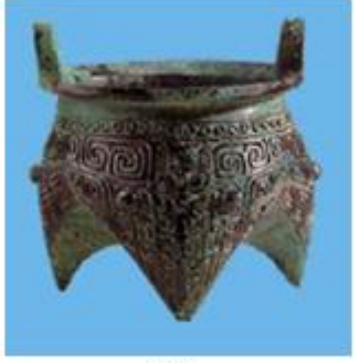

(e)

Fig. 14. Inpainting Result of "Shang Dynasty Bronze Cooking Tripod”. (a) Original Image; (b) Masked Image; (c) Criminisi Result; (d) Nan Result; (e) Our Result. 


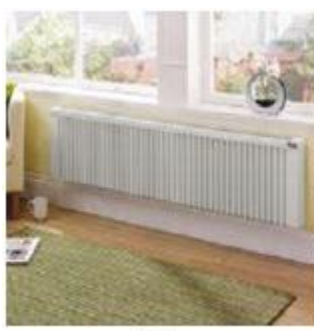

(a)

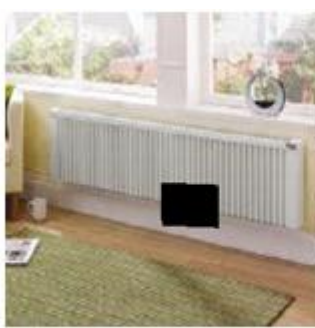

(b)

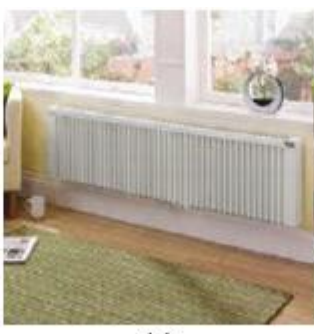

(c)

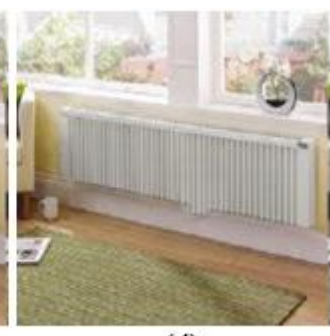

(d)

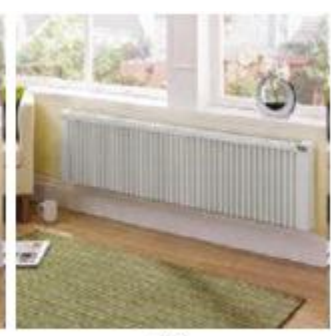

(e)

Fig. 15. Inpainting Result of "Radiator". (a) Original Image; (b) Masked Image; (c) Criminisi Result; (d) Nan Result; (e) Our Result.

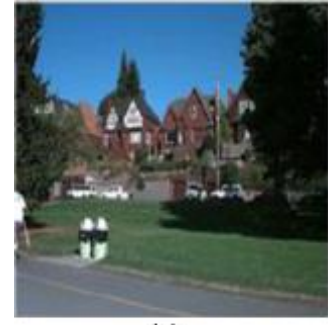

(a)

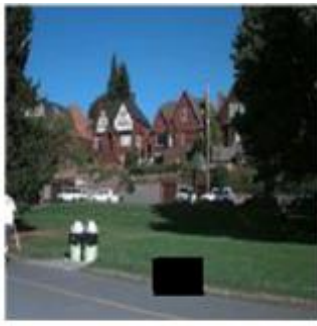

(b)

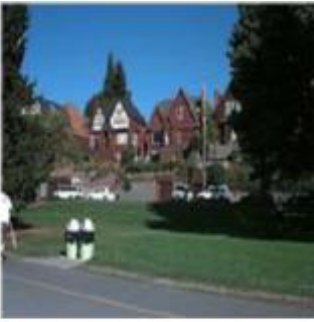

(c)

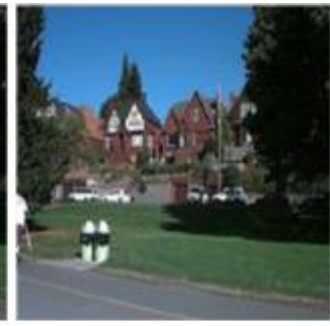

(d)

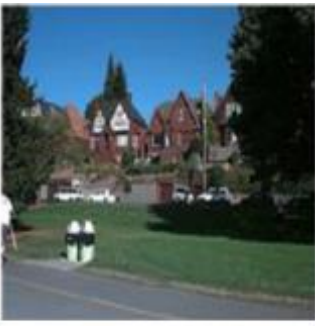

(e)

Fig. 16. Inpainting Result of "Runway". (a) Original Image; (b) Masked Image; (c) Criminisi Result; (d) Nan Result; (e) Our Result.

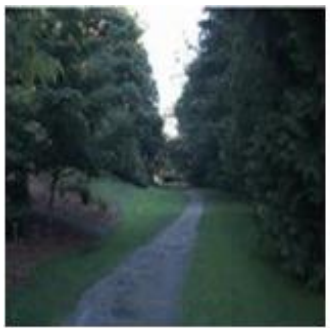

(a)

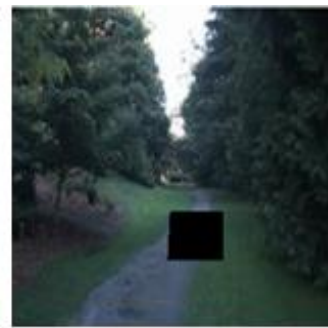

(b)

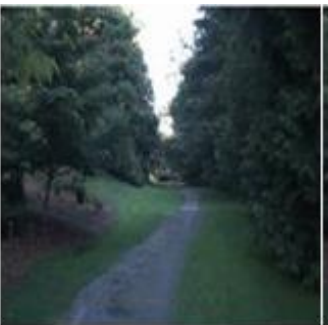

(c)

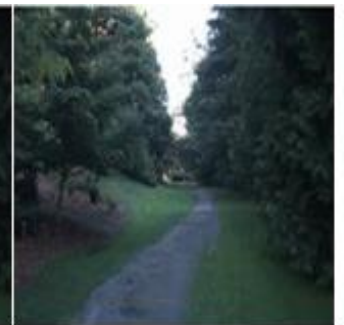

(d)

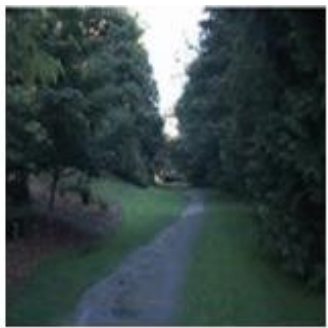

(e)

Fig. 17. Inpainting Result of "Forest". (a) Original Image; (b) Masked Image; (c) Criminisi Result; (d) Nan Result; (e) Our Result.

TABLE. IV. PSNR AND SSIM RESULTS

\begin{tabular}{|c|c|c|c|c|}
\hline & & Criminisi & Nan & Ours \\
\hline \multirow{2}{*}{ Nursery garden } & PSNR & 30,6118 & 27,9657 & 32,6820 \\
\hline & SSIM & 0,9796 & 0,9785 & 0,9804 \\
\hline \multirow{2}{*}{ Synthesis } & PSNR & 25,0545 & 42,1786 & $\infty$ \\
\hline & SSIM & 0,9838 & 0,9983 & 1,000 \\
\hline \multirow{2}{*}{ Baboon } & PSNR & 31,9447 & 35,5832 & $\mathbf{3 6 , 8 8 3 2}$ \\
\hline & SSIM & 0,9877 & 0,9918 & 0,9926 \\
\hline \multirow{2}{*}{ Boat } & PSNR & 33,2237 & 31,5973 & $\mathbf{3 3 , 5 2 0 3}$ \\
\hline & SSIM & 0,9867 & 0,9854 & 0,9886 \\
\hline \multirow{2}{*}{ Radiator } & PSNR & 43,1780 & 43,2911 & 45,6559 \\
\hline & SSIM & 0,9952 & 0,9963 & $\mathbf{0 , 9 9 7 3}$ \\
\hline \multirow{2}{*}{ Shang Dynasty bronze cooking tripod } & PSNR & 29,4801 & 28,0416 & 34,347 \\
\hline & SSIM & 0,9728 & 0,9756 & 0,9941 \\
\hline \multirow{2}{*}{ Runway } & PSNR & 44,7967 & 46,6525 & 46,9254 \\
\hline & SSIM & 0,9961 & 0,9969 & 0,9975 \\
\hline \multirow{2}{*}{ Flower } & PSNR & 30,0323 & 31,9666 & 32,3962 \\
\hline & SSIM & 0,9858 & $\mathbf{0 , 9 8 8 0}$ & 0,9874 \\
\hline \multirow{2}{*}{ Forest } & PSNR & 43,7404 & 42,0271 & 44,2778 \\
\hline & SSIM & 0,9975 & 0,9962 & 0,9981 \\
\hline \multirow{2}{*}{ Building } & PSNR & 31,0260 & 31,4830 & 32,7926 \\
\hline & SSIM & 0,9891 & 0,9889 & 0,9902 \\
\hline \multirow{2}{*}{ Landscape } & PSNR & 32,5483 & 33,9106 & 35,6248 \\
\hline & SSIM & 0,9880 & 0,9925 & 0,9942 \\
\hline
\end{tabular}


The curves obtained from our algorithm effectively show a slower decrease in the confidence term than the Criminisi one and their non-convergence at zero. Also, this same decrease can be observed in the curves of the priority function.

From Table IV, we can see that our method provides better results than the Criminisi and Nan algorithms. Indeed, the higher the PSNR and SSIM are, the better the result of inpainting is. The difference between the quality of our inpainting results and those of other methods is visible in Fig. 10 to 17.

\section{CONCLUSION}

In this work, we proposed an improvement of the inpainting algorithms based on the exemplar. We proposed a new priority function based on the weighted sum of its component terms and a new approach to calculating the data term. Our data term takes into account all the structural information of the patch and not the structural information related only to the central pixel of the patch. We have redefined the function of confidence term update to slow down its decrease and avoid its convergence towards zero. Our method generally gives better results than the literature. This is noticeable both visually and in relation to PSNR and SSIM.

We also carried out a study to select the patch size and coefficients of confidence and data terms based on the structural information of the area to be reconstructed. This study made it possible to establish a correspondence between the chosen size and the different coefficients. This correspondence is based on the structural information surrounding the area to be restored. However, this field remains open and can be improved. Thus, in our future work, we will deepen the search for the best coefficients of confidence and data terms with respect to patch size and structural information.

\section{REFERENCES}

[1] Y. Gao et al., 'Dynamic Searching and Classification for Highlight Removal on Endoscopic Image', Procedia Computer Science, vol. 107, pp. 762-767, 2017.

[2] S. M. Alsaleh, A. I. Avilés, P. Sobrevilla, A. Casals, and J. K. Hahn, 'Automatic and robust single-camera specular highlight removal in cardiac images', 2015 37th Annual International Conference of the IEEE Engineering in Medicine \& Biology Society (EMBC),pp 675-678, 2015.

[3] S. M. Alsaleh, A. I. Aviles, P. Sobrevilla, A. Casals, and J. K. Hahn, 'Adaptive segmentation and mask-specific Sobolev inpainting of specular highlights for endoscopic images', in 2016 38th Annual International Conference of the IEEE Engineering in Medicine and Biology Society (EMBC), 2016, pp. 1196-1199.

[4] S. M. Alsaleh, A. I. Aviles, P. Sobrevilla, A. Casals, and J. Hahn, 'Towards robust specularity detection and inpainting in cardiac images', in Proc. SPIE, 9786, Medical Imaging 2016: Image-Guided Procedures, Robotic Interventions, and Modeling, p. 97861Q.

[5] C. Guillemot and O. Le Meur, 'Image Inpainting: Overview and Recent Advances', IEEE Signal Processing Magazine, vol. 31, no. 1, pp. 127144, Jan. 2014.

[6] O. Le Meur and C. Guillemot, 'Super-Resolution-Based Inpainting', in Computer Vision - ECCV 2012, vol. 7577, A. Fitzgibbon, S. Lazebnik, P. Perona, Y. Sato, and C. Schmid, Eds. Berlin, Heidelberg: Springer Berlin Heidelberg, 2012, pp. 554-567.

[7] J. Zeng, X. Fu, L. Leng, and C. Wang, 'Image Inpainting Algorithm Based on Saliency Map and Gray Entropy', Arabian Journal for Science and Engineering, vol. 44, no. 4, pp. 3549-3558, Apr. 2019.
[8] S. Masnou and J.-M. Morel, 'Level lines based disocclusion', in Proceedings 1998 International Conference on Image Processing. ICIP98 (Cat. No.98CB36269), Chicago,IL,USA, 1998,vol.3,pp. 259-263.

[9] Bertalmio, G. Sapiro, V. Caselles, and C. Ballester, 'Image inpainting', in Proceedings of the 27th annual conference on Computer graphics and interactive techniques, 2000, pp. 417-424.

[10] J. Shen and T. F. Chan, 'Mathematical Models for Local Nontexture Inpaintings', SIAM Journal on Applied Mathematics, vol. 62, no. 3, pp. 1019-1043, Jan. 2002.

[11] D. TschumperlÉ, 'Fast Anisotropic Smoothing of Multi-Valued Images using Curvature-Preserving PDE's', Int J Comput Vision, vol. 68, no. 1, pp. 65-82, Jun. 2006.

[12] A. Criminisi, P. Perez, and K. Toyama, 'Region Filling and Object Removal by Exemplar-Based Image Inpainting', IEEE Transactions on Image Processing, vol. 13, no. 9, pp. 1200-1212, Sep. 2004.

[13] A. Nan and X. Xi, 'An improved Criminisi algorithm based on a new priority function and updating confidence', in 2014 7th International Conference on Biomedical Engineering and Informatics, Dalian, China, 2014, pp. 885-889.

[14] P. Yuan, X. Gong, S. Cao, J. Y. Guo, C. Y. Wang and H. M. Zou, 'A Modified Exemplar based Inpainting Algorithm', CRSSC-CWI-CGrC, 2010.

[15] Z. Hou, 'Criminisi Image Concealment Algorithm Based on Priority Function and Blocking Matching Principle', Revista Tecnica De La Facultad De Ingenieria Universidad Del Zulia, vol. 39, no. 9, pp. $203-$ 209, Dec. 2016.

[16] P. Buyssens, M. Daisy, D. Tschumperle, and O. Lezoray, 'Exemplarbased Inpainting: Technical Review and new Heuristics for better Geometric Reconstructions', IEEE Transactions on Image Processing, vol. 24, no. 6, pp. 1809-1824, Mar. 2015.

[17] Q. Zhang and J. Lin, 'Exemplar-Based Image Inpainting Using Color Distribution Analysis', Journal of Information Science and Engineering, vol. 28, no. 4, pp. 641-654, 2012.

[18] H. Ying, L. Kai, and Y. Ming, 'An Improved Image Inpainting Algorithm Based on Image Segmentation', Procedia Computer Science, vol. 107, pp. 796-801, Jan. 2017.

[19] A. A. Efros and T. K. Leung, 'Texture synthesis by non-parametric sampling', in Proceedings of the Seventh IEEE International Conference on Computer Vision, Kerkyra, Greece, 1999, pp. 1033-1038 vol.2.

[20] Y. Chi, N. He, and Q. Zhang, 'A Fast Image Restoration Method Based on an Improved Criminisi Algorithm', Journal of Computers, vol. 12, no. 6, p. 11, 2017.

[21] A. Li, Y. Li, W. Niu, and T. Wang, 'An improved criminisi algorithmbased image repair algorithm', in 2015 8th International Congress on Image \& Signal Processing (CISP), Shenyang, China,2015,pp.263-267.

[22] L.-J. Deng, T.-Z. Huang, and X.-L. Zhao, 'Exemplar-Based Image Inpainting Using a Modified Priority Definition', PLOS ONE, vol. 10, no. 10, p. e0141199, Oct. 2015.

[23] D. Lei, J. Dai-Hong, D. Bin, and J. K. Hahn, 'Improved Digital Image Restoration Algorithm Based on Criminisi', Journal of Digital Information Management, vol. 14, no. 5, p. 9, 2016.

[24] G. Ma, 'Inpainting technology in art pictures based on edge and texture feature', Journal of Interdisciplinary Mathematics, vol. 21, no. 5, pp. 1199-1203, Jul. 2018.

[25] S. Yuheng and Y. Hao, 'Image Inpainting Based on a Novel Criminisi Algorithm', arXiv:1808.04121 [cs], Aug. 2018.

[26] J. Y. Wu and Q. Q. Ruan, 'Object Removal By Cross Isophotes Exemplar-based Inpainting,' The 18th International Conference onPattern Recognition, vol. 3, pp. 810-813, 2006.

[27] X. Xi, F. Wang, and Y. Liu, 'Improved Criminisi Algorithm Based on a New Priority Function with the Gray Entropy', in 2013 Ninth International Conference on Computational Intelligence and Security, 2013, pp. 214-218.

[28] L. Yin and C. Chang, 'An effective exemplar-based image inpainting method', in 2012 IEEE 14th International Conference on Communication Technology, 2012, pp. 739-743. 